\title{
Research Critique
}

Open Access

Article History:

Received 10 April 2021

Accepted 26 April 2021

Published 1 August 2021

Available online 30 July 2021

https://doi.org/10.47544/johsk.2021.2.2.22
Corresponding Author:

Joon Young Park

jkim29@syr.edu

Department of Exercise Science

The David B. Falk College of Sport and Human Dynamics

Syracuse University, New York, USA

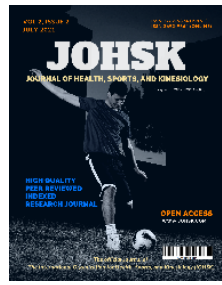

\section{Cardarine (GW501516) Effects on Improving Metabolic Syndrome}

\author{
Jeremy Park \& Joon Young Kim \\ Syracuse University, New York, USA
}

Keywords: Cardarine, GW501516, metabolic syndrome

\begin{abstract}
The present study hypothesized that treatment with GW501516 (a selective PPAR- $\delta$ agonist) lowers lipids by increasing fatty acid oxidation without adverse effects on oxidative stress. Caucasian men (age 18-50 years, $\mathrm{n}=18$ ) were randomly assigned to treatment with GW501516, GW590735, or placebo for two weeks while residing in a clinical research facility. A meal tolerance test, skeletal muscle biopsy, and blood/breath sampling were conducted. The study reported that treatment with GW501516 ameliorated multiple metabolic abnormalities associated with metabolic syndrome including oxidative stress, obesity, dyslipidemia, and insulin resistance, all while increasing fatty acid oxidation. Notably, no adverse effects were reported. However, the restricted living conditions and/or diets that the participants were subjected to likely do not resemble their normal lifestyle. Therefore, the beneficial effects of GW501516 on metabolic health observed in the study should further be investigated in a real-life setting. During participant recruitment, the use of dietary supplements were minimally considered, thereby increasing the risk for confounding effects on the metabolic parameters assessed in the study. Also, recruiting a larger and more diverse population would allow for a more detailed analysis that may benefit a broader range of people (i.e., examining the effects of GW501516 in certain ethnic groups or with/without exercise programs). Additional research on GW501516 and other PPAR- $\delta$ agonists is encouraged since it appears that this class of drugs can ameliorate multiple metabolic syndrome features. Future studies should consider additional metrics relevant to metabolic syndrome such as C-reactive protein, cortisol, and homocysteine.
\end{abstract}

\section{Introduction}

Peroxisome proliferator-activated receptors (PPARs) are a family of ligand-activated nuclear hormone receptors that are involved in the regulation of cellular metabolism. Fibrates (PPAR-a agonists) and thiazolidinediones (PPARY agonists) have been widely used to treat hypertriglyceridemia and type 2 diabetes (T2D), respectively (Tyagi et al., 201 1). In comparison to PPAR-a and PPAR-y, rather little is known about the potential of PPAR- $\delta$ in the treatment of any metabolic disorders.

Journal of Health, Sports, \& Kinesiology | ISSN 2692-9864 (Online) \& ISSN 2767-5874 (Print) | www.johsk.com 
| 2021 | Volume 2 | Issue 2 | The Journal of Health, Sports, and Kinesiology |

However, studies on mice suggest PPAR- $\delta$ agonism as a promising treatment approach for those with metabolic syndrome. Metabolic syndrome is characterized by obesity (particularly abdominal/visceral obesity), insulin resistance, hyperglycemia, dyslipidemia, and/or hypertension (Huang, 2009; Losko et al., 2016). Individuals with metabolic syndrome appear to be at higher risk for nonalcoholic fatty liver disease (NAFLD), cardiovascular disease (CVD), T2D, premature aging, and various inflammatory conditions (Al Rifai et al., 2015; Dominguez \& Barbagallo, 2016). Thus, treatment of metabolic syndrome has emerged as an essential field of clinical research. In the present study, "Activation of Peroxisome Proliferator-Activated Receptor (PPAR) Promotes Reversal of Multiple Metabolic Abnormalities, Reduces Oxidative Stress, and Increases Fatty Acid Oxidation in Moderately Obese Men", Risérus et al. examined the effects of the PPAR- $\delta$ agonist, GW501516 (also known as Cardarine), on human participants with metabolic syndrome features.

\section{Purpose}

The purpose of this study was to test the hypothesis that treatment with GW501516 lowers lipids by increasing fatty acid oxidation without adverse effects on oxidative stress (Risérus et al., 2008).

\section{Methods}

Risérus et al. conducted a two-week long, double-blind, randomized, three-parallel group study. Healthy (no sign of any clinical health disorders), but obese Caucasian men with moderate dyslipidemia were recruited. Recruits were excluded if they were smokers, regularly engaging in intense exercise, taking antihypertensive/hypolipidemic/antidiabetic medications, or if they had a history of diabetes, CVD, thyroid dysfunction, appreciably elevated liver enzymes, abnormal electrocardiogram results, and/or hyperglycemia. Inclusion criteria was a body mass index $(\mathrm{BMI})>27 \mathrm{~kg} / \mathrm{m}^{2}$, waist girth $>95 \mathrm{~cm}$, age 18-50 years, fasting plasma triglycerides (TAGs) 100-350 mg/dl, and HDL < $50 \mathrm{mg} / \mathrm{dl}$. The PPAR-a agonist, GW590735, was included to better understand the mechanisms of GW501516. Selected participants $(n=18)$ were randomly assigned to treatment with either placebo, 10 mg/day of GW501516, or 20 micrograms/day of GW590735 (n=6 for each group). Eight of the 18 selected participants fit the criteria for metabolic syndrome defined by The National Cholesterol Education Program Adult Treatment Panel III (James, 2001). Participants resided in a clinical research facility throughout the two-week study duration and were provided standardized diets. Physical exercise and alcohol consumption were inhibited. Metabolic parameters were evaluated during two visits on two consecutive days pretreatment and after 12-14 days of treatment.

Upon the first day of investigation, liver fat (\% fraction) measured by MRI and a skeletal muscle biopsy of the vastus lateralis was performed to assess mRNA expression of carnitine palmitoyl transferase 1B (CPT 1b), carnitine acyltransferase (CRAT), acyl-CoA carboxylase 2 (ACBA2), hydroxyl coenzyme dehydrogenase (HADHA), and PPAR- $\delta$. Slow and fast twitch muscle fibers were separated via laser capture microdissection (LCM). Pre- and posttreatment, a 6-hour meal tolerance test was conducted with corresponding sampling of the blood and exhaled breath. The standardized meal contained $100 \mathrm{mg}$ of [U13C]palmitate $(M+16)$ which served as a tracer for palmitate $(M+0)$. Gas chromatography and mass spectrometry (GC/MS) was used to determine fatty acid composition and isotopic enrichment (tracer/tracee ratio: TTR) of plasma nonesterified fatty acids (NEFAs) and TAGs. TRR of [13C]CO in the breath sample was used to compare postprandial fat oxidation between groups. Anthropometrics included $\mathrm{BMI}$ and weight. Plasma insulin and glucose concentrations were used to determine insulin sensitivity using the Homeostatic Model Assessment for Insulin Resistance (HOMA-IR).

In addition to TAGs and NEFAs, lipid profile was characterized by total cholesterol, LDL, HDL, and apoB. Whole-body oxidative stress was assessed via urinary F2 isoprostanes and liver enzymes measured included $Y$ glutamyl transferase ( $Y$-GT), aspartate aminotransferase (AST), and alanine aminotransferase (ALT). $\beta$-OH-butyrate was used to monitor hepatic fatty acid oxidation.

\section{Results}

Risérus et al. conducted a two-week long, double-blind, randomized, three-parallel group study. Healthy (no sign of any clinical health disorders), but obese Caucasian men with moderate dyslipidemia were recruited. Recruits were excluded if they were smokers, regularly engaging in intense exercise, taking antihypertensive/hypolipidemic/antidiabetic medications, or if they had a history of diabetes, CVD, thyroid

Journal of Health, Sports, \& Kinesiology | ISSN 2692-9864 (Online) \& ISSN 2767-5874 (Print)| www.johsk.com 
| 2021 | Volume 2 | Issue 2 | The Journal of Health, Sports, and Kinesiology |

dysfunction, appreciably elevated liver enzymes, abnormal electrocardiogram results, and/or hyperglycemia. Inclusion criteria was a body mass index (BMI) $>27 \mathrm{~kg} / \mathrm{m}^{2}$, waist girth $>95 \mathrm{~cm}$, age 18-50 years, fasting plasma triglycerides (TAGs) 100-350 mg/dl, and HDL < $50 \mathrm{mg} / \mathrm{dl}$. The PPAR-a agonist, GW590735, was included to better understand the mechanisms of GW501516. Selected participants $(n=18)$ were randomly assigned to treatment with either placebo, 10 mg/day of GW501516, or 20 micrograms/day of GW590735 (n=6 for each group). Eight of the 18 selected participants fit the criteria for metabolic syndrome defined by The National Cholesterol Education Program Adult Treatment Panel III (James, 2001).

Participants resided in a clinical research facility throughout the two-week study duration and were provided standardized diets. Physical exercise and alcohol consumption were inhibited. Metabolic parameters were evaluated during two visits on two consecutive days pre-treatment and after 12-14 days of treatment. Upon the first day of investigation, liver fat (\% fraction) measured by MRI and a skeletal muscle biopsy of the vastus lateralis was performed to assess mRNA expression of carnitine palmitoyl transferase 1B (CPTlb), carnitine acyltransferase (CRAT), acyl-CoA carboxylase 2 (ACBA2), hydroxyl coenzyme dehydrogenase (HADHA), and PPAR- $\delta$. Slow and fast twitch muscle fibers were separated via laser capture microdissection (LCM).

Pre- and post-treatment, a 6-hour meal tolerance test was conducted with corresponding sampling of the blood and exhaled breath. The standardized meal contained $100 \mathrm{mg}$ of [U13C]palmitate (M+16) which served as a tracer for palmitate $(M+0)$. Gas chromatography and mass spectrometry (GC/MS) was used to determine fatty acid composition and isotopic enrichment (tracer/tracee ratio: TTR) of plasma nonesterified fatty acids (NEFAs) and TAGs. TTR of [13C]CO in the breath sample was used to compare postprandial fat oxidation between groups. Anthropometrics included BMI and weight. Plasma insulin and glucose concentrations were used to determine insulin sensitivity using the Homeostatic Model Assessment for Insulin Resistance (HOMA-IR). In addition to TAGs and NEFAs, lipid profile was characterized by total cholesterol, LDL, HDL, and apoB. Whole-body oxidative stress was assessed via urinary F2 isoprostanes and liver enzymes measured included $Y$-glutamyl transferase ( $Y$-GT), aspartate aminotransferase (AST), and alanine aminotransferase (ALT). $\beta$-OH-butyrate was used to monitor hepatic fatty acid oxidation.

\section{Conclusion}

Two weeks of PPAR- $\delta$ activation led to beneficial effects on plasma lipid profiles, insulin sensitivity, whole-body oxidative stress, and liver fat content. mRNA analysis of the vastus lateralis suggests upregulation of fat oxidation pathways in both fast and slow muscle fibers. A strikingly large reduction in plasma NEFA was seen with GW501516 treatment and this may originate from a greater rate of fat oxidation and/or regulation of Niemann-pick C1 like-1 (NPC 1L1) by PPAR- $\delta$. Overall, it appears that GW501516 treatment would be beneficial in various ways for those with metabolic syndrome (Table 2). Limitations of the present study include a short treatment period and restricted living conditions. In summary, GW501516 appears to ameliorate numerous metabolic syndrome characteristics with minimal/absent adverse reactions.

\section{Critique}

The purpose of the study was to test the hypothesis that treatment with GW501516 lowers lipids by increasing fatty acid oxidation without adverse effects on oxidative stress. Although Risérus et al. comprehensively measured the effects of PPAR- $\delta$ on metabolic improvements in humans by using various state-of-the-art techniques such as muscle biopsy and MRI scan, several limitations should be considered to conclude the potential effects of PPAR- $\delta$ on metabolic syndrome.

As opposed to restricting the participants to a standardized diet/lifestyle, allowing participants to carry out their normal diet/lifestyle patterns would provide a more practical demonstration of the effects of GW501516. Although not statistically significant, liver fat \% tended to differ between pre- and post-treatment in the placebo group. One participant in this group exhibited $a \sim 4 \%$ increase in liver fat \% within the 2 -week period. This suggests that the restricted living conditions were significantly different from participant's regular lifestyle/diet. If attempting to examine the effects of GW501516 in a practical context, future studies should consider allowing participants to maintain their normal lifestyle/diet as much as possible. Furthermore, it should be sophisticatedly required to consider the use of dietary supplements and/or prior special dieting patterns, both which may have altered the parameters evaluated in this study, while recruiting participants.

Journal of Health, Sports, \& Kinesiology | ISSN 2692-9864 (Online) \& ISSN 2767-5874 (Print)| www.johsk.com 
Table 1. Change from Baseline to 14 days in Fasting Plasma Biochemistry, Liver Enzymes, and Urinary Isoprostanes [Adapted from Table 2 of original research study, Risérus, Ulf, et al. Diabetes, 57(2), 332-339].

\begin{tabular}{|c|c|c|c|c|}
\hline & $\begin{array}{l}\text { Placebo } \\
(n=6)\end{array}$ & $\begin{array}{c}\text { GW590735 } \\
(n=6)\end{array}$ & $\begin{array}{c}\text { GW501516 } \\
(n=6)\end{array}$ & $\begin{array}{l}\text { P-value } \\
\text { (ANOVA) }\end{array}$ \\
\hline Glucose (mmol/l) & $-.1 \pm 0.1^{*}$ & $-0.3 \pm 0.2^{*}$ & $-0.3 \pm 0.2^{*}$ & 0.25 \\
\hline Insulin (mU/I) & $1.2 \pm 1.1^{*}$ & $1.0 \pm 1.9$ & $-1.7 \pm 2.2 * \bullet \varphi$ & 0.01 \\
\hline HOMA-IR & $0.17 \pm 0.18$ & $-0.02 \pm 0.40$ & $-0.58 \pm 0.53^{*} \bullet$ & 0.006 \\
\hline NEFA $(\mu \mathrm{mol} / \mathrm{l})$ & $22 \pm 212$ & $-62 \pm 90$ & $-247 \pm 101$ & 0.01 \\
\hline Triglycerides (mmol/l) & $1.1 \pm 0.7$ & $-0.4 \pm 0.6$ & $-0.5 \pm 0.4^{*}$ & 0.16 \\
\hline Total cholesterol ( $\mathrm{mmol} / \mathrm{l})$ & $-0.3 \pm 0.5$ & $-0.6 \pm 0.4^{*}$ & $-1.0 \pm 0.7^{*}$ & 0.13 \\
\hline LDL cholesterol (mmol/l) & $-0.3 \pm 0.4$ & $-0.6 \pm 0.4^{*}$ & $-0.9 \pm 0.6^{*}$ & 0.13 \\
\hline HDL cholesterol ( $\mathrm{mmol} / \mathrm{l})$ & $-0.05 \pm 0.08$ & $0.07 \pm 0.07 *||$ & $0.003 \pm 0.07$ & 0.049 \\
\hline Aров (g/l) & $-0.06 \pm 0.09$ & $-0.12 \pm 0.06$ & $-0.20 \pm 0.15^{*}$ & 0.06 \\
\hline$\beta$-OH butyrate $(\mu \mathrm{mol} / \mathrm{l})$ & $8 \pm 27$ & $-10 \pm 37$ & $-19 \pm 23^{*}$ & 0.14 \\
\hline $\begin{array}{l}\text { Urinary F2-isoprostanes } \\
\left(\mathrm{nmol}^{-1}{ }^{*} \mathrm{mmol}^{-1} \text { creatinine) }\right.\end{array}$ & $-0.02 \pm 0.08$ & $0.05 \pm 0.10$ & $-0.10 \pm 0.05$ & 0.01 \\
\hline Y-GT (U/I) & $-3 \pm 6$ & $-5 \pm 3^{*}$ & $-8 \pm 6$ & 0.11 \\
\hline AST (U/I) & $-1 \pm 6$ & $1 \pm 2$ & $0 \pm 4$ & 0.50 \\
\hline ALT (U/I) & $-3 \pm 14$ & $-4 \pm 14$ & $-13 \pm 13$ & 0.44 \\
\hline
\end{tabular}

Notes: Data are means \pm SD.

$P$ (ANOVA) describes the overall $P$ value between groups.

${ }^{*} P<0.05$, within-group change from baseline (paired $t$ test);

$\checkmark P<0.01$, different from placebo (unpaired $t$ test);

$\checkmark P<0.01$, different from PPAR-a (unpaired $t$ test);

$P<0.05$, different from PPAR-a (unpaired $t$ test);

\& $P<0.01$, within-group change from baseline (paired $t$ test);

| $\mid P<0.05$, different from placebo (unpaired $t$ test). 
| 2021 | Volume 2 | Issue 2 | The Journal of Health, Sports, and Kinesiology |

Table 2. GW501516 Ameliorates Multiple Metabolic Syndrome-Related Abnormalities

$\begin{array}{lll}\begin{array}{l}\text { GW501516 Effect } \\ \text { Improved Lipid Profile }\end{array} & \text { Change } & \begin{array}{c}\text { Corresponding Metabolic Syndrome Features } \\ \text { Ameliorated }\end{array} \\ \text { Total/LDL Cholesterol } & \text { Decrease } \\ \text { ApoB } & \begin{array}{c}\text { Decrease } \\ \text { Decrease }\end{array} & \text { Dyslipidemia/CVD Risk } \\ \text { Increased Fatty Acid Oxidation } & \text { Increase } & \\ \text { CPTIb mRNA Expressio } & \text { Increase } & \text { Obesity/NAFLD } \\ \text { Meal-Derived Postprandial Exhalation } & \text { Decrease } & \\ \text { Liver Fat } & & \text { Type 2 Diabetes } \\ \text { Improved Insulin Sensitivity } & \text { Decrease } & \\ \text { Fasting Glucose } & \text { Decrease } & \\ \text { Fasting Insulin } & & \end{array}$

Reduced Oxidative Stress

Urinary F2-Isoprostanes Decrease

Inflammatory Diseases/Aging

In addition, a larger cohort with various ethnic backgrounds, age, and weight status is required to draw clear conclusive statement regarding the beneficial effects of Cardarine on metabolic syndrome. Moreover, future studies on potential metabolic syndrome treatments should include inflammatory markers (i.e., C-reactive protein and homocysteine) and stress indicators (i.e., cortisol) since these variables are highly implicated with metabolic syndrome (Al Rifai et al., 2015; Dominguez \& Barbagallo, 2016). The inclusion of additional metabolic syndromerelated metrics such as blood pressure may prove useful in evaluating the multifaceted benefits of PPAR- $\delta$ agonists. Later research has revealed potential oncogenic effects of GW501516 in mice which has deterred further research in humans (Mitchell \& Bishop-Bailey, 2019).

However, many of the conditions associated with metabolic syndrome, such as inflammation, appear to increase the risk of cancers (Cowey \& Hardy, 2006; Deng et al., 2016). Yet, in the present study, Cardarine was shown to reduce oxidative stress and attenuate obesity, which otherwise may activate inflammatory pathways and promote tumorigenesis (Reuter et al., 2010). Compound 1 examined by Lagu et al. is a more selective PPAR- $\delta$ agonist than GW501516 that appears to exhibit an improved safety profile (Lagu et al., 2018). Thus, further research on GW501516 and similar PPAR- $\delta$ agonists are required as some benefits may outweigh impeding risks, especially in the context of metabolic syndrome-like conditions.

\section{References}

Al Rifai, M., Silverman, M. G., Nasir, K., Budoff, M. J., Blankstein, R., Szklo, M., Katz, R., Blumenthal, R. S., \& Blaha, M. J. (2015). The association of nonalcoholic fatty liver disease, obesity, and metabolic syndrome, with systemic inflammation and subclinical atherosclerosis: The Multi-Ethnic Study of Atherosclerosis (MESA). Atherosclerosis, 239(2), 629-633.

https://doi.org/10.1016/j.atherosclerosis.2015.02.011

Cowey, S., \& Hardy, R. W. (2006). The metabolic syndrome: A high-risk state for cancer? American Journal of Pathology, 169(5), 1505-1522. https://doi.org/10.2353/ajpath.2006.051090

Deng, T., Lyon, C. J., Bergin, S., Caligiuri, M. A., \& Hsueh, W. A. (2016). Obesity, Inflammation, and Cancer. Annual Review of Pathology: Mechanisms of Disease, 11(1), 421-449.

Journal of Health, Sports, \& Kinesiology | ISSN 2692-9864 (Online) \& ISSN 2767-5874 (Print) | www.johsk.com 
| 2021 | Volume 2 | Issue 2 | The Journal of Health, Sports, and Kinesiology |

https://doi.org/10.1 146/annurev-pathol-012615-044359

Dominguez, L. J., \& Barbagallo, M. (2016). The biology of the metabolic syndrome and aging. Current Opinion in Clinical Nutrition and Metabolic Care, 19(1), 5-11. https://doi.org/10.1097/MC0.0000000000000243

Huang, P. L. (2009). A comprehensive definition for metabolic syndrome. DMM Disease Models and Mechanisms, 2(5-6), 231-237. https://doi.org/10.1242/dmm.001180

James, I. (2001). Executive Summary of the Third Report of the National Cholesterol Education Program (NCEP) Expert Panel on Detection, Evaluation, and Treatment of High Blood Cholesterol in Adults (Adult Treatment Panel III). The Journal of the American Medical Association, 285(19), 2468. www.nhlbi.nih.gov.

Lagu, B., Kluge, A. F., Goddeeris, M. M., Tozzo, E., Fredenburg, R. A., Chellur, S., Senaiar, R. S., Jaleel, M., Babu, D. R. K., Tiwari, N. K., Takahashi, T., \& Patane, M. A. (2018). Highly selective peroxisome proliferator-activated receptor $\delta$ (PPARS) modulator demonstrates improved safety profile compared to GW501516. Bioorganic and Medicinal Chemistry Letters, 28(3), 533-536. https://doi.org/10.1016/i.bmcl.2017.11.006

Losko, M., Kotlinowski, J., \& Jura, J. (2016). Long noncoding RNAs in metabolic syndrome related disorders. Mediators of Inflammation. https://doi.org/10.1155/2016/5365209

Mitchell, J. A., \& Bishop-Bailey, D. (2019). PPARß/ठ a potential target in pulmonary hypertension blighted by cancer risk. Pulmonary Circulation, 9(1), 2045894018812053. https://doi.org/10.1177/2045894018812053

Reuter, S., Gupta, S. C., Chaturvedi, M. M., \& Aggarwal, B. B. (2010). Oxidative stress, inflammation, and cancer: How are they linked? Radical Biology and Medicine, 49(11), 1603-1616.

https://doi.org/10.1016/i.freeradbiomed.2010.09.006

Risé Rus, U., Sprecher, D., Johnson, T., Olson, E., Hirschberg, S., Liu, A., Fang, Z., Hegde, P., Richards, D., Sarov-Blat, L., Strum, J. C., Basu, S., Cheeseman, J., Fielding, B. A., Humphreys, S. M., Danoff, T., Moore, N. R., Murgatroyd, P., O'rahilly, S., ... Karpe, F. (2008). Activation of Peroxisome Proliferator-Activated Receptor (PPAR) Promotes Reversal of Multiple Metabolic Abnormalities, Reduces Oxidative Stress, and Increases Fatty Acid Oxidation in Moderately Obese Men. https://doi.org/10.2337/db07-1318

Tyagi, S., Gupta, P., Saini, A., Kaushal, C., \& Sharma, S. (2011). The peroxisome proliferator-activated receptor: A family of nuclear receptors role in various diseases. Journal of Advanced Pharmaceutical Technology and Research, 2(4), 236-240. https://doi.org/10.4103/2231-4040.90879

\section{About the Authors}

\section{Joon Young Kim \\ Jeremy Park}

iD https://orcid.org/0000-0003-0448-1684

iD https://orcid.org/0000-0003-4385-1115

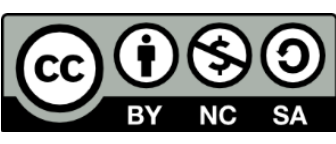

(C) 2021. This work is licensed under a CC BY-NC-SA 4.0 International license.

This is an open access article distributed under the Creative Commons Attribution License which permits unrestricted use, distribution, and reproduction in any medium, provided the original work is properly cited. 